\title{
NECESSIDADE DE INFORMAÇÃO A PAIS DE CRIANÇAS PORTADORAS DE CARDIOPATIA CONGÊNITA
}

\section{NEED OF INFORMATION FOR PARENTS WITH CHILDREN SUFFERING FROM CONGENITAL HEART DEFECTS}

\author{
Bruna Gabriela Bibancos Damas \\ Carolina Aparecida Ramos ${ }^{2}$ \\ Magda Andrade Rezende ${ }^{3}$
}

Damas BGB, Ramos CA, Rezende, MA. Necessidade de informação a pais de crianças portadoras de cardiopatia congênita. Rev Bras Crescimento Desenvolvimento Hum. 2009; 19(1): 103-113.

\section{Rsumo:}

Introdução: a equipe de enfermagem deve informar pais de crianças portadoras de cardiopatias no que diz respeito às necessidades decorrentes desta situação. As necessidades mais comuns são: informações a respeito da própria cardiopatia, promoção de atividade física, adequação da alimentação, cuidado à saúde bucal, prevenção da endocardite infecciosa, cuidados nas crises de cianose e na administração de fármacos. Objetivo: caracterizar o quanto pais de crianças portadoras de cardiopatia congênita estão informados acerca desta. Método: mapeamento sistemático de literatura nas bases MEDLINE, Cochrane, CINAHL, LILACS e SciELO, do período de 1997 a 2007 com obtenção de 17 artigos. Resultados: há necessidades pouco exploradas: cuidados por ocasião de crise de cianose, promoção de atividade física e administração de fármacos. As demais concentram a maior parte dos estudos. No entanto, mesmo nestas percebe-se que o conhecimento dos pais é incompleto e fragmentado, e isto ocorre tanto em países desenvolvidos, quanto nos em desenvolvimento. Em sua maior parte os cuidados são prestados por enfermeiros, dentistas e médicos. Programas de capacitação de pais são poucos e apenas um é descrito como exitoso. Há imperiosa necessidade de mudanças em termos de reorganização dos serviços de modo a abranger capacitação e apoio aos pais. Além disto, é necessário validar programas e protocolos de cuidados destinados a promover a capacitação destes. Tais programas devem ser flexíveis de modo a possibilitar a adaptação a cada situação clínica e aos determinantes sociais, cultural e econômico que agem sobre a família.

Palavras-chave: cardiopatias congênitas; cuidado da criança; enfermagem pediátrica, família.

"Mestre em Enfermagem Pediátrica pelo Programa de Pós-graduação em Enfermagem da Escola de Enfermagem da USP (capital). Professora do Departamento de Enfermagem Pediátrica da UNIP.

Mestranda em Enfermagem Pediátrica pelo Programa de Pós-graduação em Enfermagem da Escola de Enfermagem da USP (capital). Enfermeira no InCor (Instituto do Coração) do Hospital das Clinicas da Faculdade de Medicina da USP (Universidade de São Paulo).

3 Livre-docente. Professora Associada da Escola de Enfermagem da USP (capital) no Departamento de Enfermagem Maternoinfantil e Psiquiátrica.

Endereço para correspondência: Av. Dr. Enéas Carvalho Aguiar 419. São Paulo (capital). CEP 05403-000 E-mail: marezend@usp.br. 


\begin{abstract}
:
Introduction: the nursing staff must inform parents with children suffering from congenital heart defects regarding their needs related to this situation. The most common needs are the seven, as follows: Information related to cardiopathy, promotion of physical activities, adequate diet, care with buccal health, prevention of infectious endocarditis, care during cyanosis crisis and drug administration. Objective: to characterize how much information these parents have regarding this disorder. Method: systematic mapping on the literature in MEDLINE, Cochrane, CINAHL, LILACS and SciELO databases, from 1997 to 2007, obtaining 17 papers. Results: the following needs, cyanosis crisis, promotion of physical activities and drug administration, were not adequately studied. The majority amongst the 17 papers was concentrated on the remaining four needs. Beside this, parent's knowledge is not satisfactory and is fragmented. It happens either in developed countries as well as in the underdeveloped ones. In general, care is performed by nurses, dental physicians and physicians. Training programs for parents are scarce and only one is described as successful. Changes are mandatory in terms of reorganization of services involving training and support for parents. Besides, validation of programs and protocols of care to promote training and development is required. These programs must be flexible to allow adaptation to clinical situation and to social, cultural and economic determinant factors acting on the family.
\end{abstract}

Key words: congenital heart defects; child care; paediatric nursing; family.

\section{INTRODUÇÃO}

Cardiopatias congênitas são anomalias anatômicas no coração, que podem causar desde simples até graves alterações no funcionamento da bomba cardíaca. ${ }^{1}$ Incide em 9 (nove) entre 1000 nascidos vivos. ${ }^{2}$ Podem ser classificadas como acianóticas e cianóticas conforme desencadeiem esta situação clínica ou não.

A sobrevida de crianças portadoras de cardiopatas congênitas aumentou consideravelmente devido ao avanço tecnológico ${ }^{1}$, no entanto, o atendimento de suas necessidades (alimentação, atividade física, cuidado à saúde bucal, etc) é um tópico que merece atenção.

O nascimento de um bebê com cardiopatia pode ser uma grave situação para os pais, tanto pela situação clínica, quanto pela "morte" da criança sadia que, simbolicamente, existia antes do diagnóstico. ${ }^{3}$ Outro agravante é o tratamento, em geral longo e complexo. Por conseqüência, apoio e orientação aos pais, com vistas aos cuidados da criança são de fundamental importância.

A enfermagem pode ajudar pais e familiares na prevenção da enfermidade, o alívio ao sofrimento, assim como a proteção, a promoção, o restabelecimento da saúde. No caso de crianças cardiopatas, a enfermeira promove, juntamente com os familiares, as condições de saúde mais satisfatórias, além do melhor ambiente possível para seu crescimento e desenvolvimento. Tais intervenções podem ser terapêuticas, de apoio e aconselhamento, ou ainda de educação em saúde. ${ }^{3}$

Desta maneira, o objetivo é caracterizar o quanto pais de crianças portadoras de cardiopatia congênita estão informados acerca desta condição de saúde.

\section{MÉTODO}

Vários fatores contribuem para que sejam efetuadas poucas visitas aos serviços pediátricos de atenção à saúde, onde a detecção precoce de anomalias cardiovasculares pode trazer uma significativa diferença para a sobrevivência de crianças com lesões potencialmente passíveis de tratamento, mas que são letais se não diagnosticadas e tratadas. Por outro lado, a vivência profissional permite identificar necessidades de cuidado à criança 
cardiopata decorrentes da patologia e das alterações clínicas que esta provoca.

Durante a hospitalização estes cuidados são providos pela equipe de enfermagem, mas, após a alta, passam a ser competência de pais e familiares. São os seguintes: (1) conhecimento sobre a patologia propriamente dita, (2) promoção de atividade física, (3) promoção de alimentação e saúde bucal adequadas, (5) prevenção de endocardite bacteriana, (6) cuidado da criança por ocasião de crises de cianose e, finalmente, (7) cuidados na administração de fármacos.

Com o fim de conhecer o que a literatura científica reporta a respeito destes tópicos realizou-se mapeamento sistemático, processo que permite ordenar e classificar a literatura existente sobre determinado tema. Possibilita identificar lacunas do conhecimento, bem como contextualizá-lo. O mapeamento sistemático foi desenvolvido pela primeira vez no Centro de Coordenação do Instituto de Educação da Universidade de Londres. ${ }^{4,5}$ A busca de bibliografia foi realizada nas bases de dados MEDLINE, Cochrane, CINAHL, LILACS e Scielo. Foram usadas as palavras chave: "criança” e "cardiopatia congênita” adicionadas de "bucal”, "saúde bucal”, "atividade física”, "cianose”, "exercícios físicos”, "endocardite”, “família”, "pais”, "medicamento”, "nutrição” e "educação” em português, inglês e espanhol. Buscaram-se estudos em inglês, espanhol e português. O período delimitado foi janeiro de 1997 a abril de 2007. Os critérios de inclusão de artigos foram: tratar da necessidade de informação aos pais de crianças com cardiopatia congênita, no que diz respeito ao cuidado das situações oriundas da doença, a partir das sete necessidades apresentadas.

\section{RESULTADOS}

Foram obtidos 17 artigos, sendo 4 produzidos no Reino Unido, 2 no Canadá, 2 na
Austrália, e um em cada um dos seguintes países: Estados Unidos, Suécia, Formosa, Brasil, Alemanha, China (Hong Kong), Itália, Arábia Saudita e Espanha. Foram publicados irregularmente entre 1997 e 2002, oscilando entre nenhum e dois trabalhos por ano. Em 2003 foram publicados 4 artigos, em 2004 cinco, em 2005 um e em 2006 dois.

Com relação ao método, 11 (65\%) tinham caráter quantitativo, nenhum estudo tinha caráter unicamente qualitativo, e 6 (35\%) estudos eram mistos, utilizando tanto uma, quanto outra orientação.

A seguir os resultados são apresentados de acordo com as sete necessidades apresentadas na seção Método. Ressalta-se que uma das responsabilidades dos profissionais de saúde, e dentre estes, dos de enfermagem é informar os pais e certificar-se de que estes compreenderam no que consiste a necessidade da criança e como proceder para atendê-la.

Do ponto de vista do conhecimento dos país, o mesmo é incompleto em termos de diagnóstico, variando de praticamente $100 \%$, no caso dos pais que conheciam o nome da cardiopatia em pesquisa realizada na Austrália ${ }^{6}$, até chegar aos 59\%, referentes ao mesmo tipo de conhecimento, apresentados no estudo realizado em Hong Kong.?

Ressaltamos que conhecimento abarca desde informações sobre o diagnóstico até as conseqüências clínicas mais importantes. Assim, mesmo países desenvolvidos, como Canadá e Austrália, apresentam cifras baixas de conhecimento dos pais no que diz respeito a algum aspecto da patologia. Por exemplo, no estudo australiano ${ }^{6}$, somente $10 \%$ dos pais conseguem explicar as conseqüências fisiológicas da CIV (comunicação intraventricular). Além disto, outros artigos apresentam a mesma relação, com números intermediários, são eles: o trabalho realizado na Itália ${ }^{8}$ com 91,2\%; o realizado na Espanha ${ }^{9}, 85 \%$; no canadense ${ }^{10}, 71 \%$ e no conduzido na Arábia Saudita ${ }^{11}, 61 \%$. 
De acordo com o estudo canadense $\mathrm{e}^{10}$, o maior conhecimento acerca da cardiopatia corresponde à menor gravidade desta. Já a pesquisa realizada em Hong Kong relaciona este maior conhecimento a pais de crianças com cardiopatias cianóticas, independentemente de sua gravidade. ${ }^{7}$

Havia apenas um estudo referente à avaliação de um projeto de intervenção de enfermagem com mães, que haviam sido capacitadas durante a internação hospitalar e, após alta, recebiam atendimento domiciliar e via telefone visando resolver dúvidas referentes ao cuidado do bebê. ${ }^{12}$ Realizado em Formosa, o estudo teve avaliação muito positiva por parte das mães, que se sentiram autoconfiantes e competentes. Por outro lado, segundo estudo realizado no Reino Unido, 43\% dos pais relatavam precisar de mais informações a respeito das condições clínicas da criança. ${ }^{13}$

Noutra questão, relacionada à promoção da atividade física, os pais têm conhecimento incompleto sobre a necessidade de atividade física e, quando sabem algo, eventualmente desconhecem que a criança precisa de avaliação especializada antes de ser liberada para fazê-la. ${ }^{7,8}$ Além disto, também foi detectado que os pais não se preocupavam com a atividade física de seus filhos cardiopatas.

Outro dado apresentado nesta área e passível de discussão é o fato de pais de crianças mais velhas apresentarem maior conhecimento nesta área, quando comparados a pais de crianças mais novas. ${ }^{7}$

Ademais, a criança portadora de cardiopatia pode apresentar características clínicas que geram dificuldades para a sua alimentação, tais como problemas respiratórios e hipóxia. Além disso, tem ganho de peso menor, quando comparado ao de crianças sem a doença ${ }^{14}$, pois sua taxa metabólica é aumentada pela má função cardíaca e subsequentes aumentos das frequiências cardíaca e respiratória. ${ }^{14}$ Vale acrescentar que as mães descreve- ram esforços no sentido de ajustar a dieta das crianças a fim de que estas voltassem a ganhar a peso, tais como, uso de complementos alimentares e óleos, entre outros. Mesmo assim, os resultados ficaram aquém do necessário. ${ }^{14}$

As dificuldades respiratórias são de tal ordem, alegam as mães das crianças cardiopatas, que causam a interrupção da amamentação. Tal fato é reforçado pelos depoimentos das mães das crianças não cardiopatas, para as quais dificuldades respiratórias não foram causa de desmame. ${ }^{14}$

A maior parte das mães relatou sentir-se desencorajada a amamentar pela equipe de saúde do hospital, a qual alegava como motivos, o grande esforço da criança ao mamar no peito; a necessidade de incremento de calorias, que era motivo do uso de fórmulas; assim como a necessidade de controle da ingesta hídrica da criança, possível através da mamadeira ${ }^{15,16}$, apesar de saber que tal recomendação não procede. ${ }^{17}$

Quando se trata das crianças maiores, também pode haver dificuldade na alimentação de crianças com cardiopatias. Nas situações em que havia combinação de cardiopatia e dificuldades para a alimentação, a alimentação era mais demorada, fazendo com que os pais, relatassem cansaço e até exaustão. Esta situação causa maiores índices de ansiedade, frustração, preocupação, tensão e depressão. ${ }^{18}$ Além do prejuízo aos próprios pais, a frustração e ansiedade com as experiências de alimentar a criança cardiopata também podem se refletir na interação mãe-bebê e, posteriormente, influenciar no desenvolvimento desta criança. ${ }^{19}$

No entanto é possível minorar esta situação. Pesquisa acerca de um plano sistematizado de alta de enfermagem, realizada em Formosa, permitiu constatar que, após capacitação, as mães se sentiram mais seguras acerca dos cuidados a serem prestados a seus filhos no domicílio. ${ }^{12}$ Durante a internação praticavam os cuidados e, após a alta, eram visitadas 
e recebiam telefonemas dos pesquisadores a fim de eliminar possíveis dúvidas.

Apesar das queixas maternas, os profissionais da área de saúde têm clareza acerca da importância de suporte aos pais nesta situação tão especial. ${ }^{12,14-16}$ Assim, estudo britânico ${ }^{14}$ traz recomendações de suporte profissional para se obter uma rotina alimentar adequada para a criança cardiopata. Há também ${ }^{15,16}$ recomendações específicas: que a mãe receba apoio profissional sobre a utilização de técnicas adaptativas de aleitamento, bombas de retirada de leite, privacidade para a amamentação e locais adequados para armazenamento de leite ordenhado.

Também, a saúde bucal é fator de relevância para esta população. Os pais demonstravam pouco conhecimento sobre saúde bucal e não compreendiam a relação entre esta e alimentação, assim como também não compreendiam a relação entre o aparecimento da cárie e a escovação ou uso de flúor. ${ }^{20}$ Outros estudos reforçam esta análise de modo indireto, ao demonstrarem que os pais não relacionavam a saúde bucal da criança com a freqüência de escovação, e da consulta ao dentista. ${ }^{21,22}$

Há situações nas quais os pais sequer levam os filhos para as consultas ao dentista, embora não se saiba se isto ocorre porque o atendimento não está disponível ou porque não percebem sua importância. ${ }^{21}$ Por conseqüência, os hábitos de higiene bucal das crianças portadoras de cardiopatia são deficientes. Vinte e um por cento das crianças cardiopatas de um estudo realizado no Reino Unido ${ }^{20}$ e 5,8\% das crianças de estudo brasileiro ${ }^{21}$ nunca, ou quase nunca, escovavam os dentes.

De qualquer forma, os estudos demonstram que, de modo geral, a saúde bucal da criança cardiopata é sempre pior do que a de crianças sadias. São comuns cáries, fissuras, placa e gengivite. ${ }^{20,22}$ Há serviços norteados para a prevenção, como os descritos nos estudos realizados na Alemanha ${ }^{23}$, Suécia ${ }^{24}$ e Itália ${ }^{8}$, bem como serviço voltado para a cura, como o analisado na pesquisa realizada no Brasil. ${ }^{21}$ Segundo este, metade das crianças cardiopatas só ia ao dentista quando tinha cárie ou dor. ${ }^{21}$

Vale ressaltar que a prevenção da Prevenção da Endocardite Infecciosa (EI) abarca um corpo de informações relativamente grande, portanto desafiante. Assim, não causa admiração que os pais tenham conhecimento deficiente acerca desta ${ }^{9-11}$ : no caso do estudo realizado no Canadá ${ }^{10}$ 47\% não sabiam explicar no que a EI consistia, número que chegou a 85\%, no estudo espanhol. ${ }^{9} \mathrm{O}$ Brasil avulta com a cifra de $90 \% .{ }^{21}$ Como se vê, o mesmo fato atinge tanto países desenvolvidos, quanto um emergente.

Além do conhecimento básico sobre a EI ser deficiente, a compreensão de suas implicações também é precária. Noventa por cento dos pais brasileiros não conseguiram definir o que era, apesar de já terem ouvido a expressão e saberem que era algo perigoso para a criança. Além disto, sessenta por cento entendiam que a doença cardíaca era motivo de maior suscetibilidade a esta. ${ }^{21} \mathrm{O}$ mesmo foi obtido no estudo italiano: $72 \%$ dos pais não podiam definir endocardite, sendo que $44 \%$ entendiam que era um risco para a criança. ${ }^{8}$

Os pais tinham conhecimento deficiente sobre a profilaxia antibiótica da EI, exceto no estudo realizado na Suécia. ${ }^{24}$ A pesquisa australiana, por sua vez, reporta que $100 \%$ dos pais tinham recebido informações sobre endocardite infecciosa. No entanto, ao serem indagados, percebia-se que somente $20 \%$ tinha de fato compreendido a informação ${ }^{6}$, tal como reportado em outros estudos. ${ }^{6,18,23}$

$\mathrm{O}$ estudo brasileiro mostra um conhecimento dos pais bastante elevado (72,1\%), no entanto apenas 41,3\% sabiam da importância de uma boa saúde bucal como medida preventiva de EI. ${ }^{21}$

O conhecimento dos pais sobre a profilaxia antibiótica da EI variou desde cifras 
muito baixas ${ }^{11}$, a outras mais significativas. ${ }^{7,9}$ No entanto sabemos que este conhecimento não é dominado por todos. ${ }^{23}$

A escolaridade dos pais também demonstrou influir. Assim, a uma maior escolaridade corresponde maior nível de informação, o que, aliás, é esperado. ${ }^{7,9}$

Acerca dos cuidados em crise de cianose e na administração de fármacos, no que se refere à esta categoria, o tema foi apenas citado em estudos realizados no Reino Unido ${ }^{14} \mathrm{e}$ Formosa. ${ }^{12}$ Na pesquisa britânica ${ }^{14}$, que tinha como foco comparar padrões, dificuldades e como os pais se adaptavam às situações decorrentes da cardiopatia, foi constatado que a cianose não influenciava nas dificuldades alimentares da criança. A pesquisa realizada em Formosa ${ }^{12}$ apenas cita que o tema era abordado no treinamento e orientação às mães durante a internação.

O mesmo ocorreu na categoria cuidados na administração de fármacos, apenas citado nos estudos realizados em Formosa ${ }^{12}$ e na Itália. ${ }^{8}$ No primeiro a administração de medicamentos era um dos focos de orientação e treinamento das mães, durante o programa de alta criado pelas pesquisadoras. No entanto, não era descrito como este era aplicado. Tampouco o estudo italiano ${ }^{8}$ trazia estas informações. Neste, apenas $34,7 \%$ dos 148 pais foram capazes de informar as indicações, reações adversas e possíveis interações dos fármacos usados por seus filhos.

\section{DISCUSSÃO}

Quanto à metodologia escolhida, o mapeamento sistemático, foi útil para obter um levantamento da produção na área de informação e suporte a pais de crianças portadoras de cardiopatia congênita acerca da afecção de seus filhos. Foi possível identificar áreas que recebem mais atenção dos pesquisadores, bem como as que são quase ignoradas. O mapeamento sistemático permite direcionar recursos de pesquisas para temas que estão mais carentes de evidências. ${ }^{4,5}$

No que diz respeito aos temas que formaram as categorias, percebeu-se que há áreas melhor estudadas e outras praticamente ignoradas, tais como os cuidados em crise de cianose e na administração de fármacos. Este fato chama a atenção, uma vez que as sete áreas listadas foram arroladas devido à importância que tem para o cuidado abrangente à situação clínica da criança. Informar os pais ou responsáveis pela criança são atribuições de todos os profissionais de saúde, e também dos de enfermagem por serem os gerenciadores do cuidado. Um resultado deste tipo, no qual dois tópicos fundamentais recebem tão pouca atenção, dá margem a especular acerca das causas. Dois estudos podem ajudar nesta análise. Um, realizado na Austrália ${ }^{25}$, reporta que enfermeiros não se sentiam informados o suficiente para capacitar pacientes e familiares no uso da varfarina. O outro, estudo brasileiro ${ }^{26}$, consistiu na avaliação da orientação pós-alta de pacientes em uso de anticoagulantes e constatou que estes tinham dúvidas quanto à doença, dieta e cuidados com o uso do fármaco, resultado que apontava a necessidade de re-organização do serviço. Chama a atenção que a pesquisa foi realizada em serviço que atingiu um padrão de qualidade notório no cuidado e acompanhamento de pacientes em uso de terapia anticoagulante, o Hospital das Clínicas de Botucatu. Mesmo assim, segundo os próprios autores, percebia-se que tais pacientes, no caso adultos, tinham dificuldades. Este raciocínio também é válido para o cuidado a crianças.

Por outro lado, as outras categorias foram abordadas com maior freqüência. No entanto, isto não garante que todos os pontos importantes para uma assistência de boa qualidade tenham sido estudados. O modo de organização dos sistemas de saúde, por exem- 
plo, que varia entre os países, e nestes nas diferentes regiões e cidades, também gera diferentes necessidades de cuidados que precisam ser, cada uma, abordada e pesquisada. Este raciocínio também precisa ser feito levando em conta a inserção social, cultural, econômica do sujeito que está sendo cuidado. A interelação destes determinantes dá margem a número quase inesgotável de situações que merecem estudo. Vista a situação sob este prisma percebe-se que o número de trabalhos, como um todo, é inferior ao que se esperaria.

Outro ponto que merece atenção nesta análise é que se obteve artigos de todos os continentes, exceto África. Deve-se tomar o cuidado de lembrar que estas pesquisas não são representativas dos respectivos continentes, nem mesmo dos países em que foram realizadas, tendo em vista a pluralidade do modo de organização dos serviços de saúde, como já foi abordado. Mesmo assim, é promissor ter sido constatado que existe pelo menos um serviço em cada continente no qual se conduziu um trabalho acerca da criança com cardiopatia.

Além disto, no que diz respeito à distribuição geográfica, outro ponto chama a atenção: tanto países com melhores indicadores de desenvolvimento tem dificuldades no adequado atendimento de saúde a crianças com cardiopatias, quanto os em desenvolvimento. No entanto, há uma exceção: pesquisa conduzida na Suécia ${ }^{24}$ permitiu constatar que um dos problemas de saúde consiste no tempo do atendimento e nas orientações recebidas, considerados insuficientes pelos pais. Como se vê, um problema de gravidade bem menor quando comparado ao da pesquisa brasileira ${ }^{21}$ segundo a qual 28,8\% das crianças nunca haviam ido ao dentista, e 49,9\% só recebiam tal atendimento quando sentiam dor.

No que diz respeito a cada uma das categorias obteve-se:

Quanto à categoria conhecimento dos pais é preciso levar em conta que “conhecer” a patologia cardíaca situação significa ter e organizar uma série de informações a fim de prestar cuidado adequado à criança. Infelizmente algumas malformações cardíacas são bastante complexas, o que dificulta aos pais apropriarem-se deste conhecimento e de suas implicações. Esta categoria foi analisada a partir de oito artigos, provenientes da Austrália ${ }^{6}$, Hong Kong $^{7}$, Itália ${ }^{8}$, Espanha ${ }^{9}$, Canadá ${ }^{10}$, Arábia Saudita $^{11}$, Formosa ${ }^{12}$ e Reino Unido. ${ }^{13}$

A informação mais básica consiste no nome da patologia propriamente dita que era conhecida por $100 \%$ dos pais no estudo australiano $^{6}$ e $59 \%$ no de Hong Kong. ${ }^{7}$ Esperavase que a totalidade dos pais ou responsáveis tivessem esta informação, o que significa que os serviços não estão dando conta desta necessidade. As possíveis causas desta falta de informação não são mencionadas e precisam ser investigadas e sanadas.

Outros conhecimentos sobre a cardiopatia também são irregulares e parciais, mesmo em países desenvolvidos, o que pode indicar que os serviços de saúde precisam dar atenção a este ponto e que pesquisas precisam ser realizadas a fim de detectar os fatores determinantes desta situação. Não se pode esquecer que metodologias adequadas para ensinar pais e as próprias crianças precisam ser desenvolvidas e analisadas. Tais metodologias precisam ser desenvolvidas de modo a levar em conta especificidades de adultos e crianças, tais como, por exemplo, inserção social e cultural, bem como adequação à faixa etária, no caso de crianças.

\section{Promoção da atividade física}

As informações referentes a esta categoria foram obtidas de três estudos, um australiano ${ }^{6}$, um realizado em Hong Kong ${ }^{7}$ e um italiano. ${ }^{8}$ De acordo com o segundo ${ }^{7}$ os pais vão aprendendo por experiência, mais que por um plano de orientação intencional. Esta análise é reforçada pelo desconhecimento dos pais so- 
bre a importância de atividade física e de que a criança precisa de avaliação especializada antes de ser liberada para fazê-la. ${ }^{7,8}$ Assim, não causa admiração saber que os pais não se preocupavam com a atividade física de seus fi$l_{\text {lhos }}^{6}$, pois o panorama geral é de desconhecimento.

\section{Adequação da alimentação}

Cinco pesquisas, realizadas em Formosa $^{12}$, Reino Unido ${ }^{14}$, Estados Unidos ${ }^{15}$, Canadá ${ }^{16}$ e Austrália ${ }^{18}$ originaram esta categoria e compreendem o aleitamento materno e alimentação posterior.

Pesquisa que não fez parte do corpus deste estudo ${ }^{17}$, por estar fora do recorte temporal, já tinha reportado orientação inadequada de profissionais de saúde ao contraindicarem o aleitamento natural, sob a alegação de que a criança ficaria excessivamente cansada e teria maior gasto energético. Sem dúvida cada situação precisa ser analisada individualmente, mas o aleitamento natural não pode ser descartado a priori. Este fato demonstra a necessidade do uso de evidências, mais do que crenças como suporte das ações de cuidado. Infelizmente nossa prática clínica atual demonstra que este fato ocorre ainda nos dias atuais, necessitando de modificação urgente.

Além disto, ficou documentado ${ }^{18,19}$ estado de cansaço e até exaustão dos pais devido ao processo de alimentar seus filhos. Enfim, os trabalhos apresentam o esforço parental, especialmente materno, na promoção da alimentação e bem estar das crianças, mas com resultados aquém do necessário ${ }^{14}$, situação que exige não só reorientação do serviço de saúde, como a colaboração e ampliação de papéis dos serviços sociais e de apoio às famílias.

Por outro lado, apenas uma pesquisou focalizou a capacitação e promoção da autoconfiança materna. ${ }^{12}$ Esta experiência merece ser considerada como inspiração, com as devidas adaptações sociais, econômicas e culturais.

Outros trabalhos trazem recomendações de que a situação de alimentação da criança portadora de cardiopata seja olhada com atenção pelo profissional ${ }^{12,14-16}$, mas não a avaliação de um protocolo ou rotina de cuidado estruturado para este problema.

\section{Importância da saúde bucal}

Abordaram este assunto cinco trabalhos: um sueco ${ }^{24}$, dois britânicos ${ }^{20,22}$, um brasileiro ${ }^{21}$ e um italiano. ${ }^{8}$

Constata-se que a criança portadora de cardiopatia nunca, ou quase nunca escova os dentes, ação que deve ser imperativa no cuidado diário. ${ }^{20,21}$ Além disto, tal ocorre tanto em país emergente, como o Brasil ${ }^{21}$, quanto desenvolvido como o Reino Unido..$^{20}$ Esses problemas poderiam facilmente ser solucionados ou minimizados, caso os serviços de saúde estivessem atentos e bem organizados, uma vez que na maior parte das vezes se trata da promoção de hábito de higiene bucal.

Os estudos demonstram que a saúde bucal da criança portadora de cardiopatia é sempre pior que a de crianças sadias que os serviços odontológicos não estão alcançando tais crianças, o que é inadmissível.

\section{Prevenção da Endocardite Infecciosa}

O tema foi abordado em onze trabalhos, sendo dois produzidos no Reino Unido ${ }^{20,22} \mathrm{e} \mathrm{um}$ em cada um dos seguintes: Alemanha ${ }^{23}$, Arábia Saudita $^{11}$, Austrália ${ }^{6}$, Brasil $^{21}$, Canadá ${ }^{10}$, Espanha $^{9}$, Hong Kong ${ }^{7}$, Itália ${ }^{8}$ e Suécia. ${ }^{24}$

É importante considerar que, tal como o conhecimento acerca do que é a cardiopatia propriamente dita, a EI também é um tema desafiante, tanto para os pais quanto para os profissionais de saúde, por ser amplo e compreender inúmeros detalhes. Para os profissionais de saúde é desafio também, uma vez que necessitam organizar um relativamente largo corpo de 
informações de modo que sejam efetivamente apreendidas por pais das mais diversas formações em termos sociais, culturais e econômicos.

É elevado o número de pais que não conseguem expressar no que consiste a EI, tanto em países desenvolvidos, como Canadá ${ }^{10}$ Espanha9 ${ }^{9}$ quanto em desenvolvimento, caso do Brasil. ${ }^{21}$ A única exceção foi reportada na Suécia. $^{24}$

Assim, não causa admiração que a compreensão das implicações também seja precária, como demonstraram os estudos brasileiro $^{21}$ e italiano. ${ }^{8}$ Estes dados são preocupantes para nós, profissionais de saúde, pois, apesar dos pais entenderem que a EI era perigosa para seus filhos, tinham compreensão limitada a respeito de como evitá-la. O estudo brasileiro ${ }^{21}$, no qual só $41,3 \%$ dos pais sabiam da importância da saúde bucal na prevenção da EI, demonstra um paradoxo, pois manter a higiene bucal é relativamente fácil no comportamento cotidiano, especialmente quando se leva em conta o risco que está sendo evitado.

Também é uma preocupação para os profissionais de saúde o desconhecimento dos pais acerca da profilaxia com antibióticos. ${ }^{7,9,11,23}$

Vale a pena refletirmos sobre as implicações do estudo australiano ${ }^{6}$ no qual se reportava que a totalidade dos pais tinham recebido informações sobre EI. No entanto, 34\% não conseguiam explicar de modo lógico no que consistia a profilaxia da EI. Da mesma forma, estudo realizado no Reino Unido ${ }^{22}$, mostrava que apesar da orientação sistemática, 37\% dos 38 pais questionados, não entendiam haver relação entre a saúde bucal e o risco de endocardite. Portanto, o desafio dos profissionais consiste em criar e testar programas de capacitação de pais que realmente os tornem competentes em termos de conhecimento e ação. Logicamente os serviços também precisam estar preparados para dar o suporte necessário.

Além disto, vale ressaltar que, se na prevenção da EI os pais não conseguem reproduzir conhecimentos, é lógico supor que isto aconteça também nas outras necessidades, nas quais as informações não são sequer transmitidas (ou pelo menos, nada é informado a respeito disto).

A análise dos trabalhos aponta para o déficit de conhecimento dos pais independente da posição econômica do país de origem do estudo.

A criança portadora de cardiopatia congênita pode necessitar de cuidados bem específicos, uma vez que apresenta características fisiológicas próprias da má formação cardíaca. É inadmissível a re-introdução desta criança na família após o diagnóstico, ou, até mesmo, após a correção cirúrgica, sem oferecer aos pais uma extensa orientação, assim como uma rede de apoio que garanta suporte para o cuidado. Além disso, é necessário que se fomente os programas e protocolos de cuidados, assim como as orientações dadas em nível ambulatorial, a fim de suprir as evidentes lacunas no conhecimento dos pais.

O papel da pesquisa é evidente, cabendo-lhe validar programas e protocolos mais adequados ao cuidado da criança, lembrando que devem ser levadas em conta condições, práticas e valores da família. O papel do sistema de saúde, no fornecimento das condições necessárias para que a família obtenha o suporte indispensável também não pode ser esquecido. 


\section{REFERÊNCIAS}

1. American Heart Association (AHA) Congenital Heart Defects in Children Fact Sheet. [home page na internet]. Online resource. [acesso em 2 jul 2009]. Disponível em: http://www.americanheart.org/ presenter.jhtml?identifier $=12012$

2. American Heart Association (AHA) Diseases, conditions and treatments. [home page na internet]. Online resource. [acesso em 2 jul 2009]. Disponível em: http:// www.americanheart.org/ presenter.jhtml?identifier=3028667.

3. Wong DL. A criança com disfunção cardiovascular. In: Whaley \& Wong Enfermagem Pediátrica: elementos essenciais à intervenção efetiva. $5^{\circ} \mathrm{ed}$. Rio de Janeiro: Guanabara Koogan; 1999. P.784-5.

4. Social Care Institute for Excellence (SCIE) SCIE systematic maps: What is a systematic map? [home page na internet]. Online resource. [acesso em 5 fev 2009]. Disponível em: http://www.scie.org.uk/publications/ map/index.asp.

5. British Educational Research Association (BERA) Analysing Primary Studies in Systematic Research Synthesis: Data extraction for systematic maps [home page na internet]. Online resource. [acesso em 5 fev 2009]. Disponível em: http:// www.bera.ac.uk/analysing-primary-studiesin-systematic-research-synthesis/analysing primary-studies-in-systematic-researchsynthesis-data-extraction-for-systematicmaps/.

6. Lok SW, Menahem S. Parental perception of small ventricular septal defects in childhood. J Paediatr Child Health. 2004;40(4):180-3.

7. Cheuk DK, Wong SM, Choi YP, Chau AK, Cheung YF. Parents' undertanding of their child's congenital heart disease. Heart. 2004;90(4):435-9.

8. Chessa M, de Rosa G, Pardeo M, Negura DG, Butera G, Giamberti A, et al. What do parents know about the malformations afflicting the hearts of their children? Cardiol Young. 2005;15(2):125-9.

9. Moreno MRP, Fernandez PD, Molina JMC, López MAH, Calderón MS, Rodríguez del
Rey MMRV, et al. Prophylaxis of bacterial endocarditis in children with congenital heart disease: current status of parents' knowledge. An Esp Pediatr. 1997;47(4):389-91.

10. Bulat DC, Kantoch MJ. How much do parents know about their children's heart condition and prophylaxis against endocarditis? Can J Cardiol. 2003; 9(5):501-6.

11. Al-Jarallah AS, Lardhi AA, Hassan AA. Endocardits prophylaxis in children with congenital heart disease. A parent's awareness. Saudi Med J. 2004;25(2):182-5.

12. Yang HL, Chen YC, Mao HC, Gau BS, Wang JK. Effect of a systematic discharge nursing plan on mothers' knowledge and confidence in caring for infants with congenital heart disease at home. J Formos Med Assoc. 2004;103(1):47-52.

13. Wray J, Maynard L. The needs of families of children with heart disease. J Dev Behav Pediatr. 2006;27(1):11-7.

14. Clemente C, Barnes J, Shinebourne E, Stein A. Are infant behavioural feeding difficulties associated with congenital heart disease? Child Care Health Dev. 2001; 27(1):47-59.

15. Barbas KH, Kelleher DK. Breastfeeding success among infants with congenital heart disease. Pediatr Nurs. 2004;30(4):258-9.

16. Lambert JM, Watters NE. Breastfeeding the infant/child with a cardiac defect: an informal survey. J Hum Lact. 1998;14(3):205-6.

17. Marino BL, O’Brien P, LoRe H. Oxigen saturations durin breast and bottle feedings in infants with congenital heart disease. J Ped Nursing. 1995;19:175-79.

18. Imms C. Impact on parents of feeding young children with congenital or acquired cardiac disease. Cardiol Young. 2000;10(6):547-81.

19. Pye S, Green A. Parent education after newborn congenital heart surery. Adv Neonatal Care. 2003;3(3):147-56.

20. Saunders CP, Roberts GJ. Dental attitudes, knowledge, and health practices of parents of children with congenital heart disease. Arch Dis Child. 1997;76(6):539-40.

21. Silva DB, Souza IP, Cunha MC. Knowledge, Attitudes and status of oral health in children at risk for infective endocarditis. Int J Paediatr Dent. 2002;12(2):124-31.

22. Balmer R, Bu'Lock FA. The experiences with oral health and dental prevention of 
children with congenital heart disease. Cardiol Young. 2003;13(5):439-43.

23. Knirsch W, Hassberg D, Beyer A, Teufel T, Pees C, Uhlemann F, et al. Knowledge, compliance and practice of antibiotic endocarditis prophylaxis of patients with congenital heart disease. Pediatr Cardiol. 2003;24(4):344-9.

24. Grahn K, Wikstrom S, Nyman L, Rydberg A, Stecksen-Blicks C. Attitudes about dental care among parents whose children suffer from severe congenital heart disease: a case control study. Int J Paediatr Dent.

2006;16(4):231-8.

25. Newall F, Johnston L, Monagle P. A survey of pediatric cardiology nurses' understanding of warfarin therapy. Pediatric Cardiology 2006; 27(2): 204-8.

26. Barbosa MSS, Maffei FHA, Marin MJS Avaliação das orientações pós alta de pacientes em uso de anticoagulante oral. 2007;10(113):471-7.

Recebido em: 15/11/2008

Modificado em: 07/01/2009

Aceito em: 08/02/2009 